\title{
Aspectos estruturais do ambiente e seus efeitos nas assembléias de formigas em ambientes de floresta e bosque
}

\section{Structural environment and its effects on boards of ants in environments and forest grove}

Artigo

Original

Thais Alves Miranda ${ }^{1}$

Aline da Silva Santanna ${ }^{1}$

Original

André Barbosa Vargas ${ }^{2}$

Paper

Fábio Souto Almeida ${ }^{3}$

\section{Palavras-chave}

Riqueza de espécies

Dossel

Solo.

\section{Resumo}

Estudos sobre a biodiversidade da Mata Atlântica avançaram nos últimos anos, entretanto o conhecimento disponível sobre esse bioma ainda é restrito em abrangência geográfica e organismos analisados. Neste sentido, este estudo visou avaliar a composição, riqueza, diversidade e distribuição da fauna de formigas epigéicas (solo) e arborícolas (dossel) na Floresta da Cicuta e no Bosque do Campus do Centro Universitário de Volta Redonda - UniFOA. Para tanto foram empregadas 24 armadilhas do tipo Pitfall no solo e no dossel. Foram calculados os índices ecológicos de equitabilidade de Pielou, diversidade de Shannon-Weaver e Simpson. Para caracterizar os ambientes foram medidas a profundidade da serapilheira, a cobertura do solo, a altura e o número de árvores e arbustos, a luminosidade, a área basal, a temperatura e umidade do ar. No total, foram amostradas 25 espécies, distribuídas em quatro subfamílias. Foram encontradas seis espécies no dossel e 12 espécies no solo da Floresta da Cicuta. No Bosque UniFOA foram coletadas quatro espécies no dossel e 16 espécies no solo. Todos os atributos avaliados, neste estudo, apresentaram maiores valores para a Floresta da Cicuta, exceto a riqueza e os índices de diversidade de espécies de formigas, maiores no Bosque UniFOA. É inegável a importância da preservação dos remanescentes florestais de Mata Atlântica para a conservação da sua biodiversidade. Todavia, os resultados demonstram que a recuperação e manutenção de áreas antropizadas também podem contribuir para salvaguardar a diversidade biológica.

\footnotetext{
${ }^{1}$ Graduando em Ciências Biológicas com ênfase em Biotecnologia.

${ }^{2}$ Professor do Centro Universitário de Volta Redonda - UniFOA

${ }^{3}$ Universidade Federal Rio do Rio de Janeiro - UFRRJ. Departamento de Ciências Administrativas e do Ambiente, Instituto Três Rios.
}

Recebido em $03 / 2013$

Aprovado em 04/2013 
Studies on the biodiversity of the Atlantic Forest have advanced in recent years, however, the knowledge available on this biome is still limited in geographic range and analyzed organisms. Thus, this study aimed to evaluate the composition, richness, diversity and distribution of the epigeic ant fauna (ground) and the arboreal ant fauna (canopy) in the Cicuta Forest and in the woods of the UniFOA University Center, in the city of Volta Redonda, Brazil. Therefore, were employed 24 Pitfall traps on the grounds and in the canopy. The Pielou's Evenness Index, the Shannon-Weaver Index and the Simpson's Diversity Index were calculated. To characterize the environments were measured the depth of the leaf litter, soil cover, the height and the number of trees and shrubs, the luminosity, the basal area, temperature and air humidity. In total, 25 species were sampled, distributed in four subfamilies. Six species were found in the canopy and 12 species in the grounds of the Cicuta Forest. In the woods of UniFOA were collected four species in the canopy and 16 species in the grounds. All the attributes evaluated in this study had the highest values for the Cicuta Forest, except the richness and the diversity of ants species, the largest in the woods of UniFOA. There is no denying on the importance of the preservation of the Atlantic Forest remnants for conservation of its biodiversity. However, the results show that the recovery and maintenance of areas occupied by man can also contribute to safeguard biological diversity

\section{Introdução}

As formigas constituem uma porção importante da fauna de insetos e correspondem a cerca de $10 \%$ da biomassa animal em florestas tropicais (FITKAU; KLINGE 1973; BEATTIE; HUGHES, 2002). Além disso, são bem distribuídas dentre os mais diversos ecossistemas terrestres, exceto nas calotas polares. Do total de espécies, aproximadamente $63 \%$ nidificam e forrageiam na serapilheira (WALL; MOORE, 1999), apresentando enorme plasticidade adaptativa. Apresentam elevada riqueza e diversidade de interações com outros organismos, o que as tornam um grupo importante em processos ecológicos como a herbivoria, a ciclagem de nutrientes, a dispersão de sementes e a polinização (DEMARCO; COELHO, 2004; CUMMING, 2007).

A disponibilidade de recursos, alimentares e/ou de nidificação são fatores importantes na determinação da distribuição da riqueza e composição das assembléias de formigas (ANDERSEN, 2000). De modo geral, ambientes homogêneos abrigam menor riqueza e diversidade, apresentando uma fauna composta principalmente por espécies de hábito generalista (SOBRINHO; SCHOEREDER, 2006). Por outro lado, ambientes heterogêneos proporcionam maior disponibilidade de recursos, o que possibilita maior heterogeneidade na composição da fauna de formigas. Todavia, a complexidade ambiental em ambientes terrestres esta relacionada, principalmente à riqueza e composição de espécies vegetais (TEWS et al., 2004).

Desta forma, as formigas apresentam uma forte relação com atributos ambientais como, por exemplo, riqueza e densidade de plantas (GOMES et al., 2010a), propriedades físicas e químicas do solo (GOMES et al., 2010b) e profundidade da serapilheira (VARGAS et al., 2007) demonstrando a importância das formigas no funcionamento dos ecossistemas e, consequentemente, na conservação da biodiversidade. Assim, este estudo teve como objetivo descrever os efeitos das características do ambiente sobre a riqueza e composição da fauna de formigas em dois ambientes distintos - Floresta e Bosque.

\section{Material e Métodos}

\section{1. Área de estudo}

O levantamento da mimercofauna epigéica e arborícola foi realizado no período de agosto a setembro de 2012 em dois ambientes (Floresta da Cicuta e Bosque UniFOA).

A Floresta da Cicuta abrange parte dos municípios de Volta Redonda e Barra Mansa, situada na região sul do Estado do Rio de 
Janeiro. Esta formação florestal é caracterizada como Floresta Estacional Semidecidual Submontana (IBGE, 1992) e atualmente é protegida pelo Decreto $\mathrm{n}^{\mathrm{o}} 90.792$ de 9 de janeiro de 1985, enquadrando-se na categoria de Área de Relevante Interesse Ecológico (ARIE).

O Bosque UniFOA compreende uma área de aproximadamente 0,30 hectares, onde no século XIX funcionou a Fazenda Três Poços. Como em toda a região do Vale do Paraíba, foi uma fazenda de café e posteriormente, passando por outros ciclos de desenvolvimento agrícola do país, como a cana-de-açúcar e pecuária (DEAN, 1996). Atualmente, no bosque encontram-se árvores espaçadas, dentre elas algumas espécies como: Albizia lebbeck (Albizia), Artocarpus beterophyllus Lam. (Jaca), Citrus aurantifolia (Limoeiro), Caesalpinia echinata (Pau-brasil), Carica papaya (Mamoeiro), Crescentia cujete (Cuia ou cuieira), Chorisia speciosa (Paineira), Delonix regia (Flamboyant), Eriobotrya japônica (Ameixeira), Eugenia uniflora L. (Pitanga), Ficus benjamina (Ficus), Hibiscus sp. (Hibisco), Leucena leucocephala (Leucena), Mangifera indica (Mangueira), Morus nigra (Amoreira), Persea americana (Abacateiro), Pinus sp. (Pinus), Plectrantus barbatus Andr. (Boldo), Psidium guajava (Goiabeira), Syzygium jambolanum (Jamelão), Tecoma stans (Ipê de jardim), Terminalia catappa (Amendoeira).

De modo geral, em termos de paisagem, mesmo distantes cerca de $10 \mathrm{Km}$ em linha reta, os dois ambientes estão expostos as mesmas condições climáticas. As altitudes variam entre 300 a 500m (MONSORES et al., 1982) e o clima se enquadra no mesotérmico - Cwa de Köppen - com inverno seco e verão quente e chuvoso. A pluviosidade média anual é de $1.300 \mathrm{~mm}$, sendo o mês de fevereiro o mais quente $\left(24^{\circ} \mathrm{C}\right)$ e o mês de julho o mais frio $\left(17^{\circ} \mathrm{C}\right)$. A Geomorfologia é caracterizada por patamares colinosos aplainados, colinas estruturais isoladas e patamares tabuliformes dissecados. O solo predominante é o Podzólico Vermelho Amarelo, cuja constituição pode facilitar a ocorrência de processos erosivos (BRASIL, 1983; DIAS et al., 2001).

\subsection{Amostragem da fauna e atributos ambientais}

Nos dois estratos (solo e dossel) foram empregadas armadilhas de solo do tipo Pitfall, de acordo com o descrito em Oliveira-Santos et al. (2009). Estas armadilhas consistiram de garrafas PET (Polietileno Tereftalato) com volume de dois litros, cortadas ao meio. No seu interior foi fixado um recipiente plástico de 50 $\mathrm{ml}$, contendo uma porção de sardinha em óleo comestível e o espaço entre o recipiente PET e o recipiente de $50 \mathrm{ml}$ foi preenchido com uma solução de água e detergente.

Foram distribuídos 12 pares de armadilhas que correspondem a 24 unidades amostrais em cada ambiente, sendo 12 no solo e 12 no dossel. Estas unidades amostrais foram dispostas ao longo de um transecto de $120 \mathrm{~m}$, permanecendo ativas no campo por $48 \mathrm{~h}$. As unidades dispostas no dossel ficaram a uma altura entre 8 a $10 \mathrm{~m}$ do solo. Posteriormente, as amostras foram retiradas, rotuladas e levadas ao laboratório de Zoologia do Centro Universitário de Volta Redonda - UniFOA. Os exemplares de cada espécie, da fauna de formigas, de cada unidade amostral, foram devidamente triados, morfoespeciados e montados em via seca e depositados na coleção do próprio laboratório. Para a identificação dos gêneros foram utilizadas chaves dicotômicas (HÖLLDOBLER; WILSON, 1990; BOLTON, 1994, 2003), levando em consideração Lapolla et al. (2010). A identificação em nível espécie foi realizada quando possível com auxilio de chaves dicotômicas disponíveis em revisões taxonômicas e por meio de comparações com exemplares da Coleção Entomológica Ângelo Moreira da Costa Lima (CECL) da Universidade Federal Rural do Rio de Janeiro - UFRRJ.

Para avaliar a complexidade estrutural dos ambientes e correlacionar com a fauna de formigas encontrada foram delimitadas três parcelas de $100 \mathrm{~m}^{2}$ em cada ambiente. Dentro de cada parcela as seguintes variáveis foram mensuradas: (I) profundidade da serapilheira (PS) - medida com auxilio de uma régua graduada em centímetros; (II) temperatura do solo (TS); III) umidade relativa (UR) - ambas mensuradas com auxilio 
de um termohigrômetro digital "Termo Meter"; (IV) cobertura do solo (CS) - medida com um auxílio de um aparato de madeira com $250 \mathrm{~cm}^{2}$, sendo o mesmo divido em 25 quadrados de 10 $\mathrm{cm}^{2}$ cada. Este aparato foi colocado no solo de maneira arbitrária e a partir de então se fazia a contagem dos quadrados totalmente preenchidos por serapilheira. Foram tomadas quatro medidas para que juntas totalizassem $1 \mathrm{~m}^{2} \mathrm{e}$, posteriormente, com o número de quadrados preenchidos nas quatro medidas, foi calculado o percentual do solo coberto por serapilheira; $(\mathrm{V})$ número de árvores (Narv) - no interior de cada parcela foram contabilizadas as árvores com CAP (circunferência a altura do peito) maiores que cinco centímetros; e (VI) número de arbustos (Narb) foram contabilizados os arbustos até dois metros no interior de cada parcela.

\subsection{Análise de dados}

Os dados em porcentagem (CS - cobertura do solo por serapilheira) foram transformados para arco seno. Em seguida, estas e todas as demais variáveis foram padronizadas subtraindo sua média do valor de cada observação dividida pelo desvio padrão. Esta transformação é denominada Z - score (GOTELII; ELLISON, 2011) e padroniza a discrepância dos dados. As amostras de solo e dossel foram agrupadas com, exceção da análise de ordenação (NMDS).

Os parâmetros quantitativos avaliados para a comparação entre ambientes foram riqueza, que é o número total de espécies contidas na amostra; frequência de ocorrência, que é o número total de registros da espécie nas amostras; além dos índices ecológicos de equitabilidade de Pielou, diversidade de ShannonWeaver e Simpson (MAGURRAM, 1988).

$\mathrm{Na}$ análise de variância, as parcelas foram usadas como repetição e a média para cada parâmetro ecológico (riqueza, diversidade e equitabilidade) foi calculada para cada parcela. O mesmo foi feito para as variáveis ambientais - porcentagem de cobertura do solo por serapilheira (CS); profundidade da serapilheira (PS), luminosidade (LUM), circunferência a altura do peito (CAP) e número de árvores (N_Arv).

Para verificar a similaridade entre as parcelas de amostragem, foi elaborada uma matriz de similaridade baseada no índice de
Bray-Curtis, para cada parcela. Em seguida, foi confeccionada uma ordenação com algoritmo de agrupamento pela média (KREBS, 1989). As amostras foram ordenadas por escalonamento multidimensional não-métrico (NMDS), utilizando a distância fornecida pelo índice de Bray-Curtis.

\section{Resultados}

Foram amostradas 25 espécies de formigas no total, sendo seis no dossel e 12 no solo da Floresta da Cicuta; quatro no dossel e 16 espécies no solo do Bosque UniFOA. As espécies estão distribuídas nas subfamílias Dolichoderinae, Formicinae, Ecitoninae e Myrmicinae, sendo esta última a mais representativa. Do total de espécies, apenas sete foram comuns aos dois ambientes (Floresta e Bosque). Dentre os gêneros destacam-se Camponotus (Mary 1861), Pheidole (Westwood 1839) e Solenopsis (Westwood 1840) com maior riqueza.

No ambiente de floresta as espécies Pheidole sp. 2 e Pheidole sp. 3 foram as mais frequentes. Já no ambiente de bosque foram Solenopsis sp. 1 e Pheidole sp. 1. As espécies reconhecidamente bem adaptadas a ambientes antropizados como Camponotus crassus Mayr (1862), Camponotus sericeiventris (Guérin-Méneville 1838), Wasmannia auropunctata (Roger 1863) e Tapinoma melanocephalum (Fabricicius 1793) foram registradas somente no ambiente de bosque em armadilhas de solo. As espécies Camponotus sp. 2, Camponotus sericeiventris, Camponotus sp. 4, Crematogaster evallans (Forel 1907), Pheidole sp. 1, Pheidole sp. 5 e Solenopsis invicta (Buren 1972) foram espécies que ocorreram nos dois ambientes (Tabela 1).

Todos os atributos avaliados neste estudo apresentaram maiores valores para o ambiente de floresta (Floresta da Cicuta), exceto a riqueza e os índices de diversidade de espécies, maiores no bosque (Bosque UniFOA). A análise de variância revelou diferenças significativas para a riqueza de espécies de formigas e a diversidade de Simpson. Já para os índices de diversidade de Shannon e a Equitabilidade de Pielou estas diferenças não foram detectadas. Esta mesma análise revelou diferenças 
significativas para a profundidade de serapilheira, umidade relativa e número de árvores e arbustos. Porém, mesmo sendo maiores no ambiente de floresta a temperatura e cobertura do solo não foram significativamente diferentes (Tabela 2).

Quanto à similaridade da fauna, a análise de ordenação (MDS), com base na frequência de ocorrência das espécies nas amostras, apontou uma clara distinção na composição de espécies, com a formação de dois grandes grupos. Por outro lado, os estratos dossel e solo não apresentaram um padrão distinto. Todavia, apesar dos resultados indicarem uma composição de espécies similar entre o solo e dossel, observa-se uma leve tendência ao agrupamento das amostras de mesmo estrato para cada ambiente (Figura 1).

Tabela 1: Lista das espécies coletadas na Floresta da Cicuta e no Bosque UniFOA em agosto de 2012 com a frequência de ocorrência em cada ambiente Amostrado $(\mathrm{CD}=$ Floresta da Cicuta/Dossel; $\mathrm{CS}=$ Floresta da Cicuta/Solo; BD = Bosque UniFOA/Dossel; e BS = Bosque UniFOA/Solo), riqueza, espécimes exclusivas, diversidade de Simpson e Shannon e Equitabilidade para cada ambiente.

\begin{tabular}{|c|c|c|c|c|}
\hline & $\mathrm{CD} / \%$ & $\mathrm{CS} / \%$ & BD $/ \%$ & $\mathrm{BS} / \%$ \\
\hline \multicolumn{5}{|l|}{ Dolichoderinae } \\
\hline Linepithema neotropicum & - & - & - & $1 / 8,4$ \\
\hline Tapinoma melanocephalum & $1 / 8,4$ & - & - & - \\
\hline \multicolumn{5}{|l|}{ Formicinae } \\
\hline Brachymyrmex sp. 1 & - & $2 / 16,6$ & - & - \\
\hline Camponotus crassus & - & - & - & $1 / 8,4$ \\
\hline Camponotus sericeiventris & $1 / 8,4$ & $2 / 16,6$ & - & $2 / 16,6$ \\
\hline Camponotus sp. 1 & $1 / 8,4$ & $2 / 16,6$ & - & - \\
\hline Camponotus sp. 2 & $1 / 8,4$ & $1 / 8,4$ & - & $2 / 16,6$ \\
\hline Camponotus sp. 3 & - & $1 / 8,4$ & - & - \\
\hline Camponotus sp. 4 & $1 / 8,4$ & - & - & $2 / 16,6$ \\
\hline Camponotus sp. 5 & - & - & - & $1 / 8,4$ \\
\hline Camponotus melanoticus & - & - & $1 / 8,4$ & - \\
\hline \multicolumn{5}{|l|}{ Ecitoninae } \\
\hline Labidus praedator & - & - & - & $1 / 8,4$ \\
\hline \multicolumn{5}{|l|}{ Myrmicinae } \\
\hline Cardiocondyla obscurior & - & - & $1 / 8,4$ & - \\
\hline Crematogaster evallans & - & $1 / 8,4$ & $1 / 8,4$ & $1 / 8,4$ \\
\hline Pheidole gertrude & - & $1 / 8,4$ & - & - \\
\hline Pheidole sp. 1 & - & $2 / 16,6$ & - & $3 / 25$ \\
\hline Pheidole sp. 2 & $1 / 8,4$ & $6 / 50$ & - & - \\
\hline Pheidole sp. 3 & - & $4 / 33,4$ & - & - \\
\hline Pheidole sp. 4 & - & - & - & $1 / 8,4$ \\
\hline Pheidole sp. 5 & - & $1 / 8,4$ & - & $1 / 8,4$ \\
\hline Solenopsis sp. 1 & - & - & $2 / 16,6$ & $3 / 25$ \\
\hline Solenopsis invicta & - & - & - & $1 / 8,4$ \\
\hline Solenopsis sp. 3 & - & $2 / 16,6$ & - & $1 / 8,4$ \\
\hline Solenopsis sp. 4 & - & - & - & $1 / 8,4$ \\
\hline Wasmannia auropunctata & - & - & - & $2 / 16,6$ \\
\hline Riqueza por área & 6 & 12 & 4 & 16 \\
\hline Espécies exclusivas & 1 & 4 & 2 & 8 \\
\hline Diversidade de Simpson & 0,42 & 0,82 & 0,22 & 0,84 \\
\hline Diversidade de Shannon & 0,63 & 1,73 & 0,33 & 1,87 \\
\hline Equitabilidade de Pielou & 0,67 & 0,95 & 1,00 & 0,98 \\
\hline
\end{tabular}


Tabela 2: Resultados da análise de variância (ANOVA) com os valores de Tukey para os atributos ecológicos e ambientais avaliados na amostragem da fauna de formigas na Floresta da Cicuta e no Bosque UniFOA.

\begin{tabular}{l|c|c}
\hline & \multicolumn{2}{|c}{ AMBIENTES (Floresta da Cicuta x Bosque UniFOA) } \\
\hline ATRIBUTOS ECOLÓGICOS & $\mathrm{F}$ & $\mathrm{P}$ \\
\hline Riqueza & 12,100 & 0,025 \\
\hline Equitabilidade (J') & 0,034 & 0,862 \\
\hline Shannon Weaver (H') & 6,690 & 0,061 \\
\hline Simpson (1/D) & 8,047 & 0,047 \\
\hline ATRIBUTOS AMBIENTAIS & $\mathrm{F}$ & $\mathrm{P}$ \\
\hline PS & 61,916 & 0,001 \\
\hline TS & 1,078 & 0,358 \\
\hline UR & 28,145 & 0,006 \\
\hline CS & 4,542 & 0,100 \\
\hline Narv & 28,176 & 0,006 \\
\hline Narb & 85,662 & 0,001 \\
\hline
\end{tabular}

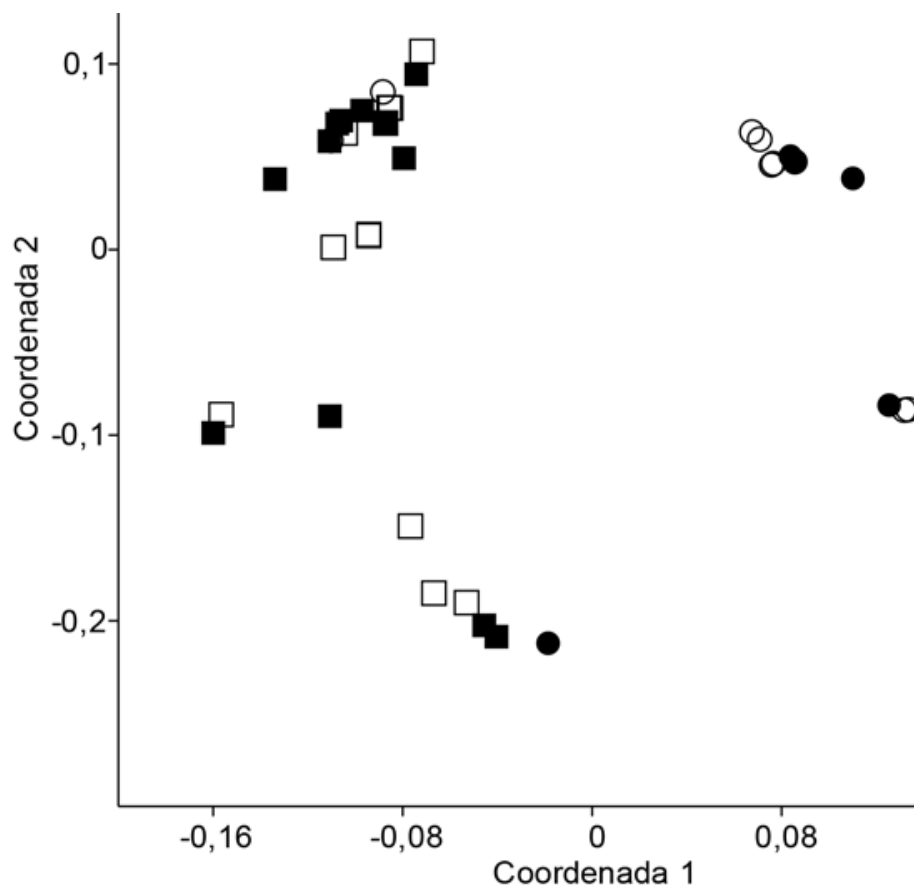

Figura 1: Ordenação das amostras pelo escalonamento multidimensional não-métrico (NMDS). (Quadrados brancos - Floresta da Cicuta/Dossel; Quadrados pretos - Floresta da Cicuta/Solo; Círculos brancos - Bosque UniFOA/Dossel e Círculos prestos - Bosque UniFOA/Solo).

\section{Discussão}

De modo geral, a composição de espécies encontrada neste estudo reflete o padrão observado para outras áreas de Mata Atlântica, exceto a maior riqueza de espécies de Camponotus, gênero com frequência em ambientes de clima sazonal (LEAL, 2003; CORRÊA et al., 2006). Todavia, as espécies desse gênero, incluindo C. crassus e C. sericeiventris, são frequentes em estudos envolven- do amostragens na vegetação (BATTIROLA et al., 2005; YAMAMOTO; DEL CLARO, 2008; FAGUNDES et al., 2010). No mesmo sentido, sendo amostrada em diversos ambientes, tanto no solo quanto sobre a vegetação, $W$. auropunctata é uma espécie exótica com hábitos onívoros, dominante e agressiva (SOUZA et al., 2008). Outra espécie bem comum em ambientes antrópicos e que pode veicular microorganismos patogênicos é $T$. melanocephalum, amostrada no bosque UniFOA. 
A subfamília Myrmicinae foi a mais representativa, pois é dominante em diversos ecossistemas brasileiros, tanto em número de gêneros quanto de espécies (SANTOS et al., 1999, MACEDO, 2004, CORRÊA et al., 2006, VARGAS, et al., 2007; ALBUQUERQUE; DIEHL, 2009, ILHA et al., 2009; MARTINS, et al., 2011). Esta predominância se deve à sua diversificação de hábitos alimentares e de nidificação, já que esta subfamília apresenta espécies onívoras, predadoras e consumidoras de líquidos e de fungos (HÖLLDOBLER; WILSON, 1990).

Vale ressaltar a ausência, neste estudo, das subfamílias Ponerinae e Pseudomyrmecinae. Na subfamília Ponerinae a maior parte das espécies forrageiam e nidificam na serapilheira. Já as espécies da subfamília Pseudomyrmecinae em sua maioria forrageiam e nidificam na vegetação (HÖLLDOBLER; WILSON, 1990; FERNÁNDEZ 2003). Ambas as subfamílias são frequentemente amostradas em estudos que utilizam armadilhas do tipo pitfall no solo e dossel (OLIVEIRA-SANTOS et al., 2009).

A disponibilidade de recursos alimentares e/ou de nidificação está relacionada com a heterogeneidade do ambiente (PIANKA, 1994; LASSAU; HOCHULI, 2004). Ambientes mais heterogêneos geralmente ofertam maior diversidade de recursos para as formigas, o que é um fator determinante para a coexistência das espécies, por disponibilizar uma gama maior de nichos ecológicos que ambientes simplificados e reduzir a competição. Neste sentido, a riqueza e a diversidade de espécies em ambientes tropicais está fortemente relacionadas a riqueza e a composição da flora (TEWS et al., 2004). No entanto, a riqueza e a diversidade de espécies de formigas observadas neste estudo não corroboraram a esta prerrogativa, já que foram constatadas diferenças significativas entre os ambientes, apontando maior riqueza e diversidade para o ambiente de bosque, que é justamente o ambiente mais homogêneo. Entretanto, Marinho et al., (2002) também obteve resultados semelhantes ao encontrar maior riqueza de espécies para um ambiente menos complexo.

Por outro lado, os atributos ambientais apontam maior heterogeneidade para a Floresta da Cicuta. Nesta floresta observou-se maior número de árvores e arbustos que originam uma boa cobertura do solo e, uma camada de serapilheira mais diversificada ao contrário do observado no bosque UniFOA. Neste bosque predomina uma paisagem mais aberta, com menor densidade de árvores, áreas com gramado e solo exposto e, uma camada de serrapilheira bastante homogênea e inexistente em alguns pontos.

A Floresta da Cicuta mesmo protegida por lei desde 1985, vem ao longo dos anos, passando por intervenções como fogo, caça, pecuária e retirada de madeira, principalmente nos arredores. Atualmente, a entrada esta proibida, exceto para atividades de cunho científico no sentido de coibir tais intervenções antrópicas e priorizar sua recuperação. Além disso, este remanescente florestal é de suma importância para a manutenção da espécie Alouatta guariba clamitans Cabrera, 1940 (bugio-ruivo), pois abriga uma das suas últimas populações na região do Vale do Paraíba (ALVES; ZAÚ, 2005). Contudo, os resultados obtidos aqui não corroboraram a hipótese de que ambientes mais heterogêneos abrigam maior riqueza e diversidade de espécies de formigas como demonstrado por outros autores (NAKAMURA et al., 2003; VARGAS et al., 2007; MARTINS et al., 2011; FREIRE et al., 2012).

Por outro lado, o bosque mesmo mais homogêneo em termos estruturais, apresenta algumas variáveis não mensuradas neste estudo e que podem estar relacionadas ao padrão observado. Como exemplo, pode-se citar a disponibilidade de alimentos provenientes da alimentação dos micos e pássaros realizada diariamente pelos funcionários no bosque, os resíduos de alimentos deixados pelos estudantes em lixeiras dispostas no Bosque UniFOA, além da presença de espécies vegetais frutíferas e nativas também podem proporcionar maior disponibilidade de recursos a mirmecofauna.

É inegável a importância da preservação dos remanescentes florestais de Mata Atlântica para a conservação da sua biodiversidade. Todavia, os resultados demonstram que e recuperação e manutenção de áreas antropizadas, como o bosque aqui estudado, também podem contribuir para salvaguardar a diversidade biológica. 


\section{Agradecimentos}

Ao Centro Universitário de Volta Redonda - UniFOA pelo auxílio financeiro e autorização do espaço para pesquisa; Aos funcionários do ICMBio unidade responsável pela ARIE - Floresta da Cicuta e a Prof. ${ }^{\text {a Kelly }}$ Carla A. de S. Borges e a discente Mariane Silva pela identificação das espécies vegetais.

\section{Referências}

ALBUQUERQUE, E. Z.; DIEHL, E. Análise faunística das formigas epígeas (Hymenoptera, Formicidae) em campo nativo no Planalto das Araucárias, Revista Brasileira de Entomologia , v. 53, p. 398-403, 2009.

ALVES, S. L.; ZAÚ S. A. A importância da área de relevante interesse ecológico da floresta da Cicuta (RJ) na conservação do Bugio-Ruivo (Alouatta guariba clamitans Cabrera, 1940), Revista Universidade Rural, v. 25, p. 41-48, 2005.

ANDERSEN, A. N. A global ecology of rainforest ants: functional groups in relation to environmental stress and disturbance. p. 2534. In: D. Agosti; J. D. Majer; L. E. Alonso \& T. R. Schultz. Ants: Standard methods for measuring and monitoring biodiversity. Washington, Smithsonian Institution Press, 2000. 280 p.

BATTIROLA, L. D.; ADIS, J.; MARQUES.; M. I.; SILVA, F. H. O. Comunidade de artrópodes associada à copa de Attalea phalerata Mart. (Arecaceae), durante o período de cheia no Pantanal de Poconé, Neotropical Entomology, v. 36, p. 640-651, 2007.

BEATtIE, A. J.; HUGHES, L. Ant-plant interactions, In: C.M. Herrera; O. Pellmyr, Plant-animal interactions: An evolutionary approach. Oxford, Blackwell Science, 2002. p. 211-235.

BOLTON, B. Identification guide to the ant genera of the world. Cambridge: Harvard University Press, 1994, 222 p.
BOLTON, B. Synopsis and classification of Formicidae. Memoirs of the American Entomological Institute, v. 71, p. 1-370, 2003.

BRASIL. Levantamento de recursos naturais, Rio de Janeiro/Vitória. RADAMBRASIL, v. 31, p. 23-24, 1983.

CORREAA, M. M.; FERNANDES, W. D.; LEAL, I. R. Diversidade de formigas epigéicas (Hymenoptera: Formicidae) em capões do Pantanal Sul Matogrossense: relações entre riqueza de espécies e complexida de estrutural da área, Neotropical Entomology, v. 35, p. 724-730, 2006.

CUMMING, G. S. Global biodiversity scenarios and landscape ecology. Landscape Ecology, v. 22, p. 671-685, 2007.

DEAN D.; MARCUS L. F.; BOOKSTEIN F. L. Chi square test of biological space curve affinities. In: Marcus LF, Corti M, Loy A, Naylor GJP, Slice DE, eds. Advances in Morphometrics. Plenum, p. 235-251, 1996.

DE-MARCO JR., P.; COELHO, F. M. Services performed by the ecosystem: Forest remnants influence agricultural cultures' pollination and production. Biodiversity and Conservation, p. 1245-1255, 2004.

DIAS, J. E.; GOMES, O. V. O.; GOES, M. H. B. Áreas de risco de erosão do solo: uma aplicação por geoprocessamento. Floresta e Ambiente, v. 8, p. 1-10, 2001.

FAGUNDES, R.; TERRA, G.; RIBEIRO, S. P.; MAJER, J. D. O Bambu Merostachys fischeriana (Bambusoideae: Bambuseae) como habitat para formigas de floresta tropical montana. Neotropical Entomology, v. 39. n. 6, p. 906-911, 2010.

FERNÁNDEZ， F. Introducción a las hormigas de la región Neotropical. Instituto Humboldt, Bogotá, 2003, 424p.

FITKAU, E. J.; H. KLINGE. On biomass and trophic structure of the Central Amazonian Rain Forest ecosystem. Biotropica, v. 5, p. 2-14, 1973. 
FREIRE, C. B.; OIVEIRA, G. V.; MARTINS, F. R. S.; SOUZA , L. E. C.; LAUCAU, L. S. R.; CORRÊA, M. M. Riqueza de formigas em áreas preservadas e em regeneração de caatinga arbustiva no sudoeste da Bahia, Revista Brasileira de Biociências, v. 10, p. 131-134, 2012.

GOMES, J. P.; IANNUZZI, L.; LEAL, I. R. Resposta da Comunidade de Formigas aos Atributos dos Fragmentos e da Vegetação em uma Paisagem da Floresta Atlântica Nordestina. Neotropical Entomology, v. 39, p. 898-905, 2010a.

GOMES, J. B. V.; BARRETO, A. C.; MICHEREFF, M. F.; VIDAL, W. C. L.; COSTA, J. L. S.; OLIVEIRA-FILHO, A. T.; CURI, N. Relações entre atributos do solo e atividade de formigas em restingas. Revista Brasileira de Ciências do Solo, v. 34, p. 6778, $2010 b$.

GOtelli, N. J.; ELLISON, A. M. Princípios de estatística em ecologia. Ed Artmed, 611p. 2011.

HÖLlDOBLER, B.; WILSON, E. O. The Ants. Harvard University Press, Cambridge, 732p., 1990.

IBGE. Manual técnico da vegetação brasileira. Rio de Janeiro, IBGE, Manuais técnicos em geociências, 166p. 1992.

ILHA, C.; LUTINSKI, J. A.; PEREIRA, D. V. M.; GARCIA, F. R. M. Riqueza de formigas (Hymenoptera, Formicidae) da Bacia da Sanga Caramuru, município de Chapecó- SC. Biotemas, v. 22, p. 95-105, 2009.

KREBS, C. J. Ecological Methodology. New York: Harper Collins, 1989.

LAPOLLA, J. S., BRADY, S. \& SHATTUCK, S. Phylogeny and taxonomy of the Prenolepis genus-group of ants (Hymenoptera: Formicidae). Systematic Entomology, v.35, p. 118-131, 2010.

LASSAU, S. A.; HOCHULI, D. F. Effects of habitat complexity on ant assemblages. Ecography, v. 27, p. 157-164, 2004.
LEAL, I. R. Diversidade de formigas em diferentes unidades de paisagem da Caatinga, Editora da Universidade Federal de Pernambuco, p. 435-460, 2003.

MACEDO, L. P. M. Diversidade de formigas edáficas (Hymenoptera: Formicidae) em fragmentos da Mata Atlântica do Estado de São Paulo. Tese (Doutorado em Entomologia) - Escola Superior de Agricultura Luiz de Queiroz. Universidade de São Paulo, Piracicaba, 2004.

MAGURRAM, A. E. Ecological Diversity and its Measurement. Cambridge. 179 p., 1988.

MARINHO, C. G. S.; ZANETTI, R.; DELABIE, J. H. C.; SCHILINDWEIN, M. N.; RAMOS, L. S. Diversidade de formigas (Hymenoptera: Formicidae) da serapilheira em eucaliptais (Myrtaceae) e área de Cerrado de Minas Gerais. Neotropical Entomology, v. 3, n. 2, p. 187-195, 2002.

MARTINS, L.; ALMEIDA, F. S.; MAYHÉNUNES, A. J.; VARGAS, A. B. Efeito da complexidade estrutural do ambiente sobre as comunidades de formigas (Hymenoptera: Formicidae) no município de Resende, RJ, Brasil. Revista Brasileira de Biociências, v. 9, p. 174-179, 2011.

MONSORES, D. W.; BUSTAMANTE, J. G. G.; FEDUlLO, L. P. L.; GOUVEIA, M. T. J. Relato da situação ambiental com vistas à preservação da área da Floresta da Cicuta, Relatório técnico, 17 p., 1982.

NAKAMURA, A.; PROCTOR, H.; CATTERALL, C. P. Using soil and litter arthropods to assess the state of rainforest restoration. Ecological Management and Restoration, v. 4, p. 20-28, 2003.

OLIVEIRA-SANTOS, L. G. R.; LOYOLA, R. D.; VARGAS, A. B. Canopy traps: A Technique for Sampling Arboreal Ants in Forest Vertical Strata. Neotropical Entomology, v. 38, p. 691-694, 2009. 
PIANKA, E. Evolutionary ecology. New York: Harper Collins College Publishers, p. 594, 1994.

SOUZA, E.; FOLLETT, P. A.; PRICE, D. K.; STACY, E. A. Field Suppression of the Invasive Ant Wasmannia auropunctata (Hymenoptera: Formicidae) in a Tropical Fruit Orchard in Hawaii. Journal Economic Entomological, v. 101, n. 4, p. 1068-1074, 2008.

SOBRINHO, T. G.; SHOEREDER, J. H. Edge and shape effects on ant (Hymenoptera: Formicidae) species richness and composition in forest fragments. Biodiversity and Conservation, v. 16, p. 1459-1470, 2006.

TEWS, J.; U. BROSE, V.; GRIMM, K.; TIELBORGER, M.C.; WICHMANN, M.; SCHWAGER.; JETSCH, F. Animal species diversity driven by habitat heterogeneity/ diversity: The importance of keystone structures, Journal of Biogeography, v. 31, p.79-92, 2004.
VARGAS, A. B.; MAYHE-NUNES, A. J.; QUEIROZ, J. M.; ORSOLON, G. S.; FOLLYRAMOS, E. Efeito de fatores ambientais sobre a mirmecofauna em comunidade de restinga no Rio de Janeiro, RJ. Neotropical Entomology, v. 36, p. 28-37, 2007.

YAMAMOTO, M. \& DEL-CLARO, K. Natural history and foraging behavior of the carpenter ant Camponotus sericeiventris Guérin, 1838 (Formicinae, Campotonini) in the Brazilian tropical savanna. Acta Ethologica, v.11, p. 55-65, 2008.

WALL, D. H. \& MOORE, J. C. Interactions underground, BioScience, v. 49, p.109-117, 1999. 